\title{
ACE2 in tumor cells and tumor vasculature: Negligible intercellular transfer from cancer cells into endothelial cells
}

\author{
Zhi-Jie Liu ${ }^{1, a}$, Yan Mei ${ }^{1, a}$, Jiang-Li Lu ${ }^{2, a}$, Jia-Bin Lu ${ }^{2, a}$, Yun Cao ${ }^{2, a}$, Mu-Yan Cai ${ }^{2}$, Li-Sheng Zheng ${ }^{1}$, \\ Ming-Dian Wang ${ }^{1}$, Li-Xia Peng ${ }^{1}$, Yang Li ${ }^{3}$, Bi-Jun Huang ${ }^{1}$, Jin-Ping Yun ${ }^{2}$, and Chao-Nan Qian ${ }^{1,4}{ }^{*}$ \\ ${ }^{1}$ State Key Laboratory of Oncology in South China, Collaborative Innovation Center for Cancer Medicine, \\ Sun Yat-sen University Cancer Center, Guangzhou 510060, PR China \\ 2 Department of Pathology, Sun Yat-sen University Cancer Center, Guangzhou 510060, PR China \\ 3 Department of Pathology, Guangzhou Concord Cancer Center, Guangzhou 510555, PR China \\ 4 Department of Radiation Oncology, Guangzhou Concord Cancer Center, Guangzhou 510555, PR China
}

Received 1 March 2021, Accepted 6 July 2021, Published online 2 August 2021

\begin{abstract}
Cancer patients are more susceptible to severe coronavirus disease 2019 (COVID-19), which is caused by severe acute respiratory syndrome coronavirus 2 (SARS-CoV-2) infection. Angiotensin-converting enzyme 2 (ACE2) is the functional host receptor for SARS-CoV-2 entering into human cells. Bioinformatics' analyses have revealed that ACE2 is upregulated in some cancer cells. In the present study, we evaluated ACE2 protein expression levels in several common malignancies compared to non-cancerous normal tissues. ACE2 expression was elevated in colorectal adenocarcinoma, pancreatic adenocarcinoma, gastric adenocarcinoma, and papillary renal cell carcinoma cancer. Yet, it was suppressed in chromophobe renal cell carcinoma, testicular germ cell tumors, and papillary thyroid carcinoma. Two tumor tissue microarrays were used to evaluate the prognostic value of ACE2 expression in patients with gastric adenocarcinoma, and colorectal adenocarcinoma without COVID-19. No significant survival benefit was found for patients with overexpression of ACE2 in the tumor. In the tumor vasculature, ACE2 expression was observed in only 54\% of the tumor micro-vessels. Using an in vitro co-culture of endothelial cells and tumor cells overexpressing fusion protein ACE2-red fluorescent protein, we did not observe any clear and convincing intercellular transfer of ACE2 from cancer cells into endothelial cells. In summary, alteration of ACE2 expression was found in common malignancies, but there is no evidence of intercellular transfer of ACE2 from cancer cells to endothelial cells.
\end{abstract}

Key words: ACE2, SARS-CoV-2, COVID-19, Endothelial cells, Gastric cancer, Pancreatic cancer, Intercellular exchange.

\section{Introduction}

Angiotensin-converting enzyme 2 (ACE2) is gene mapped on the $\mathrm{X}$ chromosome and contains 18 exons. This gene codes an 805 amino acid type I transmembrane glycoprotein essential in the development of hypertension [1]. ACE2 and its genetic variants are secreted and circulate in the bloodstream [2]. Bioinformatics' analyses have revealed elevated expression of ACE2 in colorectal adenocarcinoma, renal papillary cell carcinoma, pancreatic adenocarcinoma, gastric adenocarcinoma, and lung adenocarcinoma [3]. Moreover, elevated expression of ACE2 in cancer cells has been reported to be correlated with better patient prognosis in uterine corpus endometrial carcinoma, renal papillary cell carcinoma, and breast cancer $[4,5]$.

*Corresponding author: qianchn@sysucc.org.cn

${ }^{a}$ These authors contributed equally to this work
Another important role of ACE2 is that it is a receptor for severe acute respiratory syndrome coronavirus 2 (SARSCoV-2) infection, which caused the recent COVID-19 pandemic. Given the fact that cancer patients infected with SARS-CoV-2 develop severe manifestations and possess a higher mortality rate in contrast with non-cancerous patients $[6,7]$, it is reasonable to ask whether ACE2 expressed by cancer cells is shared to normal cells, particularly blood vessel endothelial cells, which are critical for tumor development as well as SARS-CoV-2 infection [8].

The exchange of cell surface receptor proteins among cells in the immune system has been well documented [9]. The intercellular exchange of proteins among endothelial cells and cancer cells has also been observed $[10,11]$. Moreover, intercellular exchange of transferrin receptors from cancer cells to surrounding fibroblasts has been reported [11]. In the present study, we evaluate ACE2 protein expression in different 
cancers and explore whether ACE2 can transfer from cancer cells into endothelial cells.

\section{Methods}

\section{Tissue samples and clinicopathological data}

A total of 1175 paraffin-embedded tumor tissues and 1058 matched non-cancer tissues were collected from the Department of Pathology at Sun Yat-sen University Cancer Center (SYSUCC) with the approval of the Institutional Review Board. Tissues included malignancies from the thyroid, stomach, pancreas, colon and rectum, kidney (types 1 and 2 papillary renal cell carcinoma, chromophobe renal cell carcinoma), and testis. Among these tissues, 420 cases of colorectal cancer and 475 cases of gastric cancer were constructed into tissue microarrays with tissue cores $1 \mathrm{~mm}$ in diameter, and these tissues with sufficient patient follow-up data have been previously used and reported [12, 13].

\section{Immunohistochemistry (IHC) analysis}

ACE2 immunohistochemical (IHC) staining was performed using rabbit anti-human ACE2 Abs (Abcam, ab272690) with a working dilution of 1:50. The staining intensity and proportion of positive cells were assessed as previously reported [14-16]. Briefly, the staining intensity was scored $0,1,2$, or 3 for a negative read, and the staining intensity was low, medium, or high. The proportion of positive cells were recorded as $0 \%$, $1-10 \%, 11-50 \%, 50-80 \%$, and $81-100 \%$, which corresponded to scores $0,1,2,3$, and 4 respectively. IHC scores were determined by multiplying the intensity score and the positive cell proportion score ranging from 0 to 12 . Two pathologists independently assessed the IHC scores, and the final IHC staining value was the mean value from the two pathologists' scores. Patients were divided into the low expression or high expression group using the median IHC staining value as the cutoff value.

\section{Cell culture}

Human gastric cancer cell line MGC803 and pancreatic cancer cell line Capan-2 were maintained in Dulbecco's Modified Eagle's Medium (DMEM) supplemented with 10\% fetal bovine serum (FBS). Human microvascular endothelial cells (HMVEC) were cultured in an EGM-2MV Bullet Kit medium (CC-3202, Lonza) supplemented with 10\% FBS. All cells were grown in a $37{ }^{\circ} \mathrm{C}$ humid incubator supplemented with $5 \% \mathrm{CO}_{2}$.

\section{Virus production, infection, and stable transfection}

To generate a fusion protein of human ACE2 and red fluorescent protein (RFP), the hACE2 overexpressing lentivirus plasmid (pLV[Exp]-mCherry:T2A:Puro-EF1A $>$ hACE2 [NM_021804.3], plasmid ID: VB200706-2658vya) and the control plasmid (pLV[Exp]-mCherry/Puro-EF1A $>$ Stuffer300, plasmid ID: VB180713-1052gdp) with mCherry and puromycin dual reporter gene overexpression were purchased from VectorBuilder (Guangzhou 510663, PR China) and the package plasmids pLP1, pLP2, and pVSVG were from System Biosciences (Palo Alto, CA 94303, USA). For virus production, 4-5 $\times 10^{5} 293 \mathrm{~T}$ cells were seeded in a 6-well plate overnight to reach a density of 70-80\% confluency at the time of transfection. The hACE2 overexpressing lentivirus plasmid or control plasmid were co-transfected with the three package plasmids into 293T cells using TransInTM EL Transfection Reagent (TransGen Biotech, Beijing 100192, PR China) according to the product manual of the supplier. Four to six hours after transfection, the cell culture medium was changed with $4 \mathrm{~mL}$ fresh DMEM and supplemented with 20\% FBS and incubated for $48 \mathrm{~h}$. The media were then collected and centrifuged at $1250 \mathrm{rpm}$ for $5 \mathrm{~min}$ to collect the supernatant for harvesting the virus. The cancer cells MGC803 and Capan- 2 were seeded at an appropriate density to attach overnight for virus infection. The media were then discarded and changed with a virus containing a supernatant with $6 \mu \mathrm{g} / \mathrm{mL}$ polybrene. Eight hours post-infection, the cell culture media were changed with a fresh medium supplemented with 10\% FBS and incubated for $24 \mathrm{~h}$. Puromycin $(2 \mu \mathrm{g} / \mathrm{mL})$ was then added to the cells for stably infected cell selection. The stably infected cells were further isolated using fluorescence-activated cell sorting with MoFlo (Beckman-Coulter, Brea, CA 92821-6232).

\section{Co-culture of human cancer cells and HMVEC}

HMVEC cells $\left(2 \times 10^{4}\right)$ were seeded into a 96-well plate. After $12 \mathrm{~h}, 2 \times 10^{4}$ MGC803 or Capan- 2 cells, which had been stably transfected with lentiviral plasmid overexpressing ACE2-RFP fusion protein, were added into the wells. After $24 \mathrm{~h}$, the co-cultured cells were observed by high content screening microscopy (ImageXpress Micro Confocal, Molecule Devices, USA) and images were taken every $15 \mathrm{~min}$ for the next $24 \mathrm{~h}$.

\section{Statistical analysis}

Graphpad prism software version 8.0 (GraphPad Software, San Diego, CA, USA) was used for the statistical analyses. The two-sided Wilcoxon signed-rank test was used to compare ACE2 staining values between tumor tissues and normal tissues. Survival analysis was performed using the KaplanMeier methods and the log-rank test. A $p<0.05$ was considered significant.

\section{Results}

\section{ACE2 expression profiles in human tumors}

Bioinformatics' analyses have shown that ACE2 is aberrantly expressed in many types of tumors [3]. Our IHC analyses showed that ACE2 protein expression was significantly increased in colorectal adenocarcinoma, pancreatic adenocarcinoma, gastric adenocarcinoma, and papillary renal cell carcinoma cancer tissues compared with their corresponding normal tissues. It was significantly decreased in chromophobe 

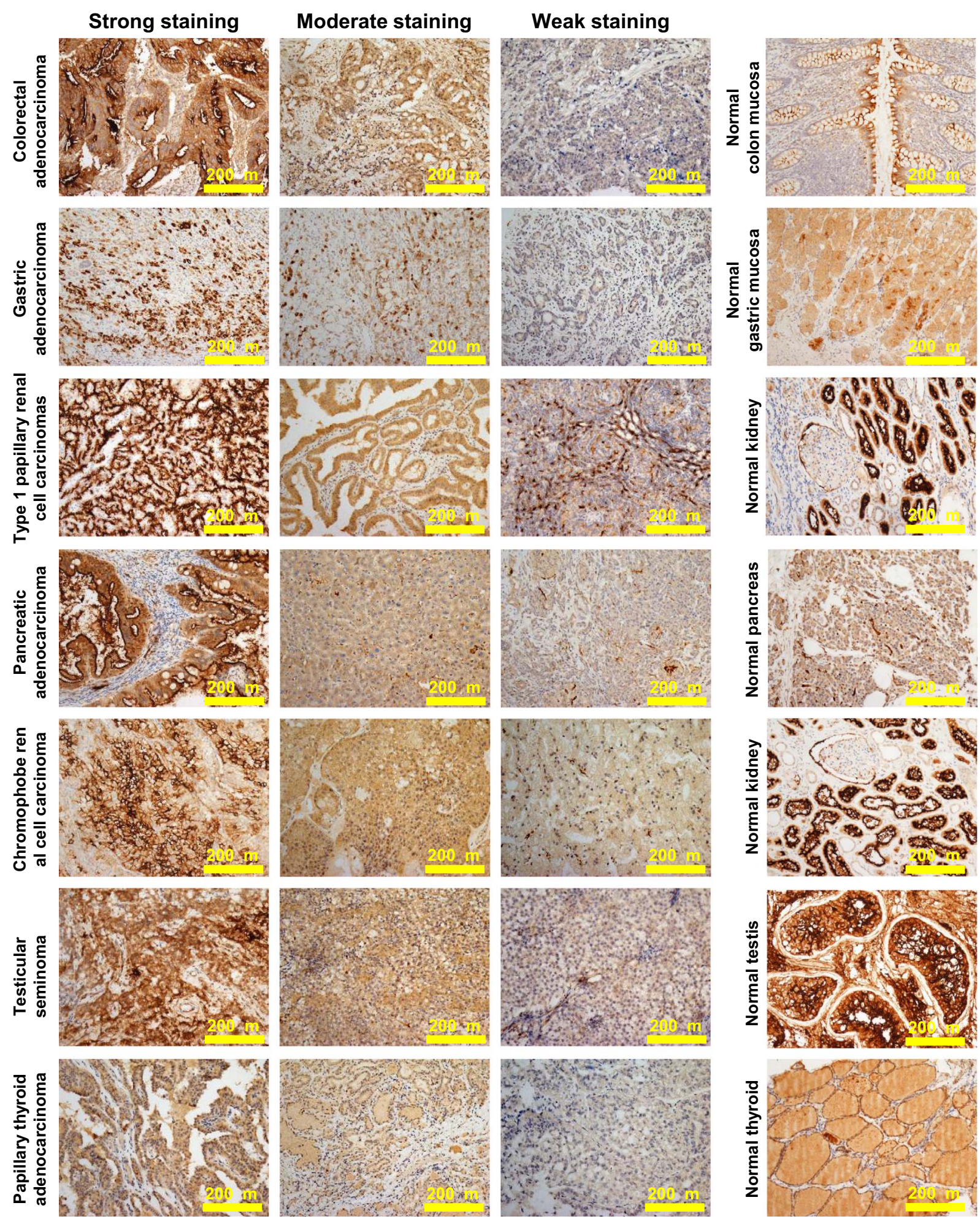

Figure 1. ACE2 protein expression in tumors and corresponding normal tissues. IHC staining of ACE2 was conducted in tumor tissues surrounding normal tissues including two tissue microarray sets of gastric cancer and colorectal cancer. ACE2, stained in brown, can be seen in the cytoplasm and cellular membrane.

renal cell carcinoma, testicular germ cell tumors, and papillary thyroid carcinoma (Figures 1-2). Survival analyses showed that although gastric cancer and colorectal cancer patients had elevated ACE2 expression in their tumor tissues seemed to possess better overall survival, the difference was not statistically significant (Figure 3). 


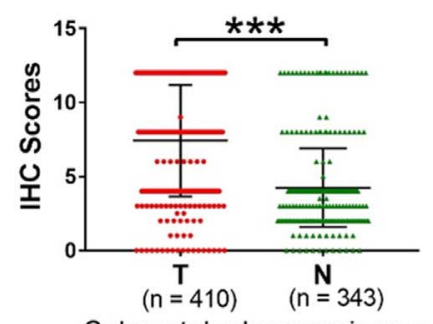

Colorectal adenocarcinoma
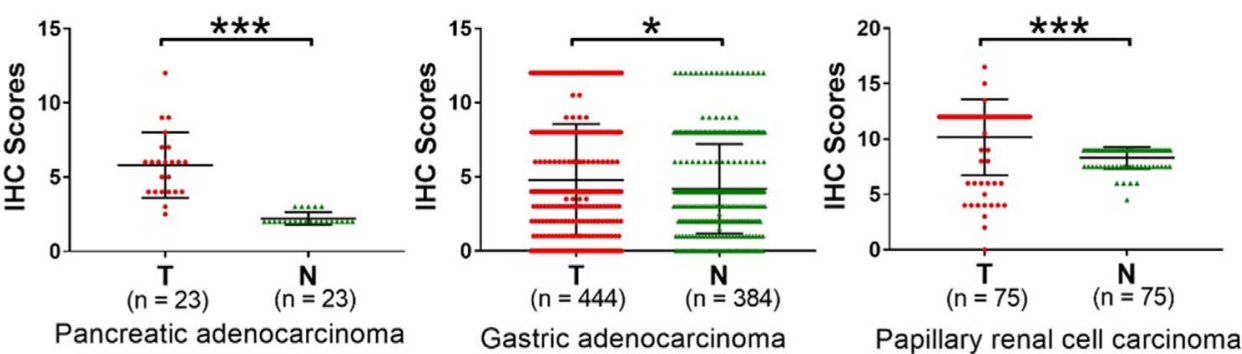

Papillary renal cell carcinoma

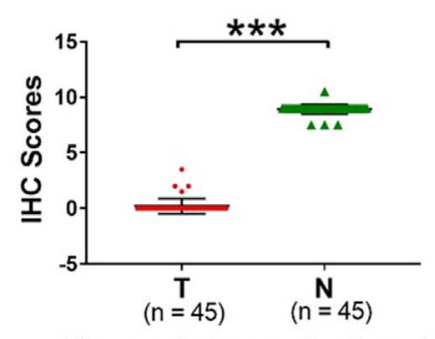

Chromophobe renal cell carcinoma

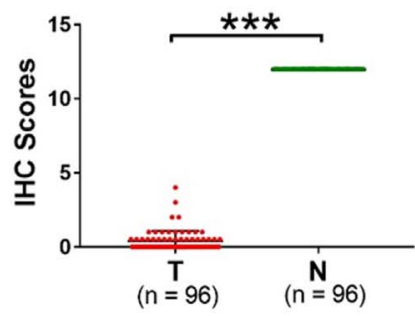

Testicular seminoma

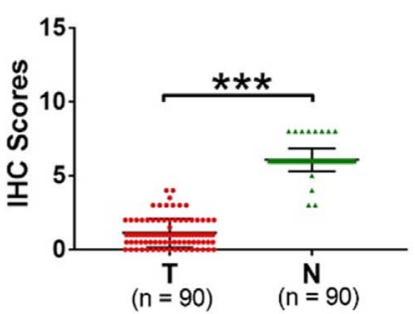

Papillary thyroid adenocarcinoma

Figure 2. Alteration of ACE2 protein expression in common malignancies. By semi-quantifying ACE2 protein expression, elevated expression was found in colorectal adenocarcinoma, pancreatic adenocarcinoma (matched tissues), gastric adenocarcinoma, and papillary renal cell carcinoma (matched tissues). While reduced expression was found in matched tissues of chromophobe renal cell carcinoma, testicular seminoma, and papillary thyroid adenocarcinoma. T, tumor tissues; $\mathrm{N}$, normal tissues. $*<0.05 ; * * *<0.01$.
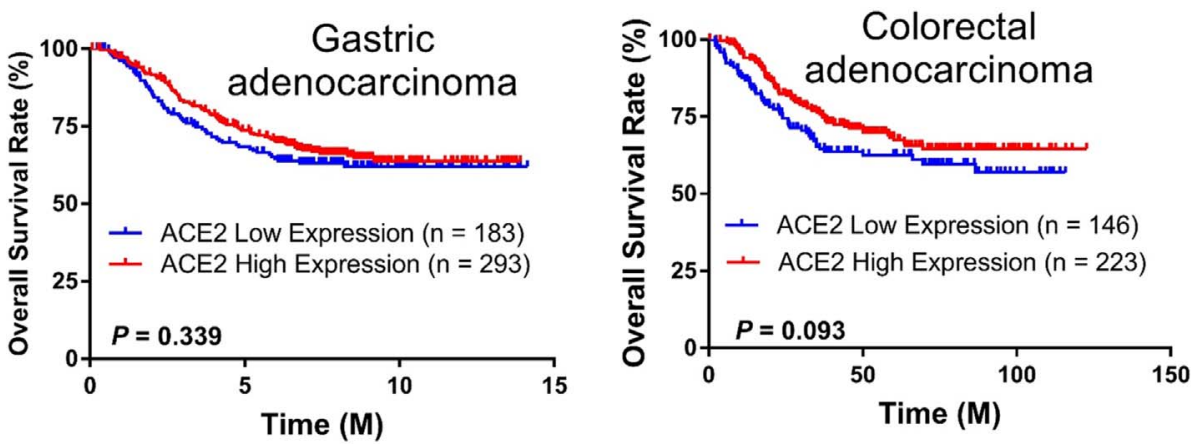

Figure 3. Alteration of ACE2 expression in the tumor does not affect patients' overall survival rates in patients with colorectal adenocarcinoma or gastric adenocarcinoma. The median value of ACE2 expression evaluated by IHC (3.5 for the gastric cancer cohort and 6 for the colorectal cancer cohort) was used as a cutoff value to separate patients into a low versus high expression group. Although the patients with elevated ACE2 expression in their tumors had favorable survival potential, no statistical significance was found in either cancer cohort.

\section{ACE2 expression on tumor vasculature is limited to a portion of the tumor blood vessels}

ACE2 expression in the endothelial cells of the tumor vasculature was observed in only $54 \%$ of the tumor blood vessels (Figure 4). Thus, ACE2 expression is negligible in some tumor vasculature, suggesting that this receptor is either replaceable or a surrogate ACE2 could be achieved by intercellular exchange of this protein.

\section{ACE2 transfer from tumor cells into endothelial cells was not observed}

To validate the existence of ACE2 intercellular transfer from tumor cells into endothelial cells, we co-cultured HMVEC cells with human gastric cancer cells MGC803 or pancreatic cancer cells Capan-2 for $24 \mathrm{~h}$. The absence of ACE2 transfer from cancer cells into endothelial cells was confirmed (Videos 1-4). However, this in vitro experiment needs to be validated using a long-term culture or an in vivo experiment to exclude the possibility of ACE2 transferring from tumor cells into endothelial cells.

\section{Discussion}

Since December 2019, SARS-CoV-2 has spread worldwide. Evidence shows that SARS-CoV-2 uses ACE2 as its receptor $[17,18]$. It seems that cancer patients have a higher risk of SARS-CoV-2 infection and poorer outcomes than 
A

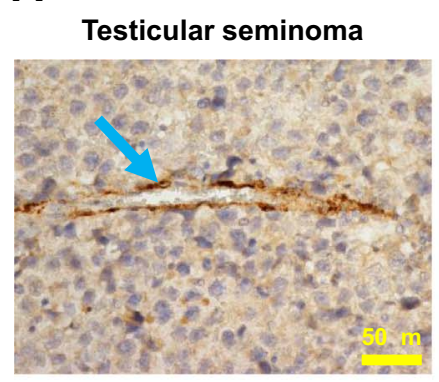

Pancreatic adenocarcinoma

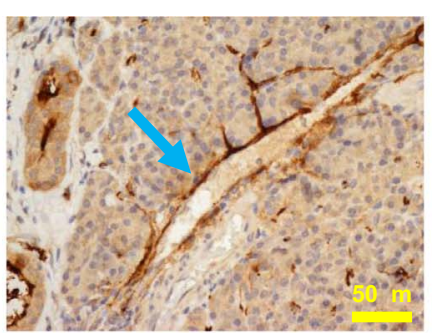

Chromophobe renal

cell carcinoma

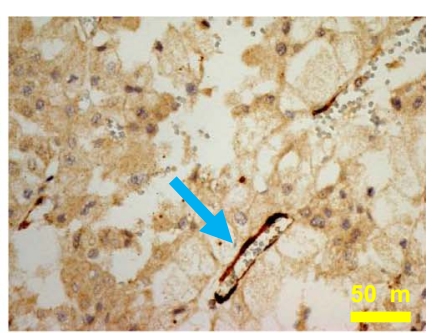

B

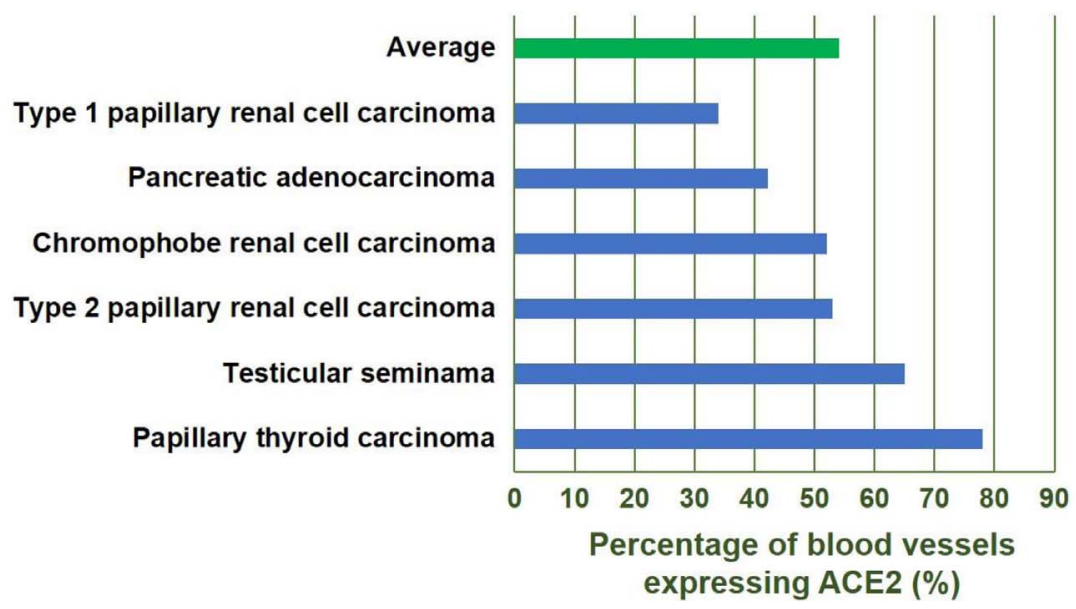

Figure 4. ACE2 expression in the tumor vasculature. (A) Examples of ACE2 staining in the tumor vasculature. (B) For each cancer type, a total of 100 tumor microvessels were counted from 10 different tumor tissues, except for pancreatic adenocarcinoma, in which a total of 90 tumor microvessels were counted in 9 tumor tissues. Different cancer types had a varying percentage of ACE2 positive tumor microvessels, resulting in an average of only 54\% tumor microvessels expressing ACE2.

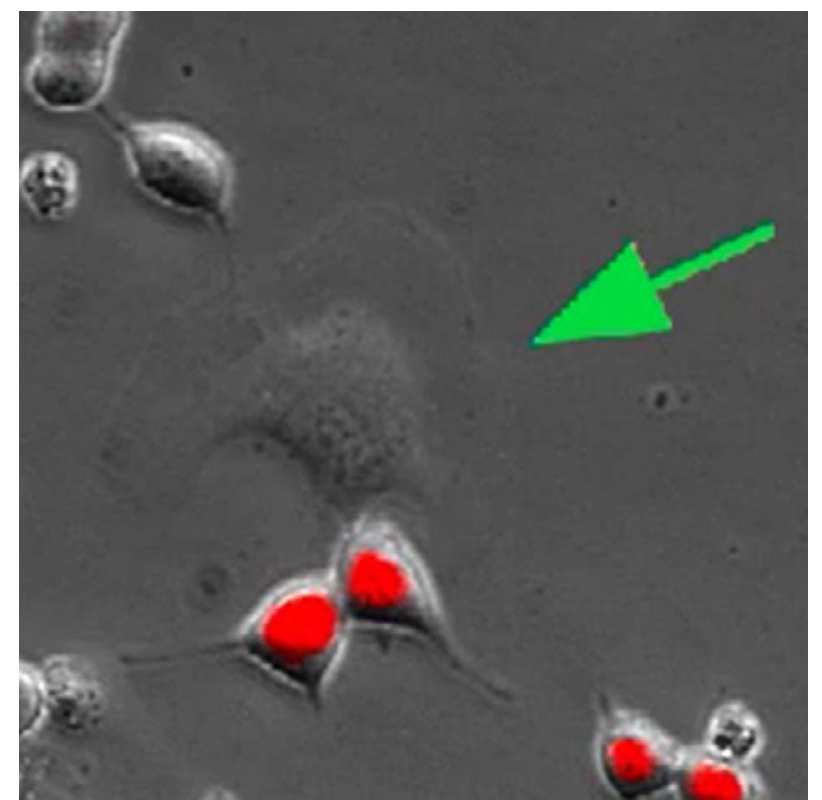

Video 1. Co-culturing MGC803 human gastric cancer cells with HMVEC cells. A time-lapse video monitoring the movement of the fusion protein ACE2-RFP (red) in cancer cells. Intercellular ACE2-RFP transfer from cancer cells into endothelial cells (green arrow) was not observed. https://vcm.edpsciences.org/10.1051/vcm/20210003\#V1

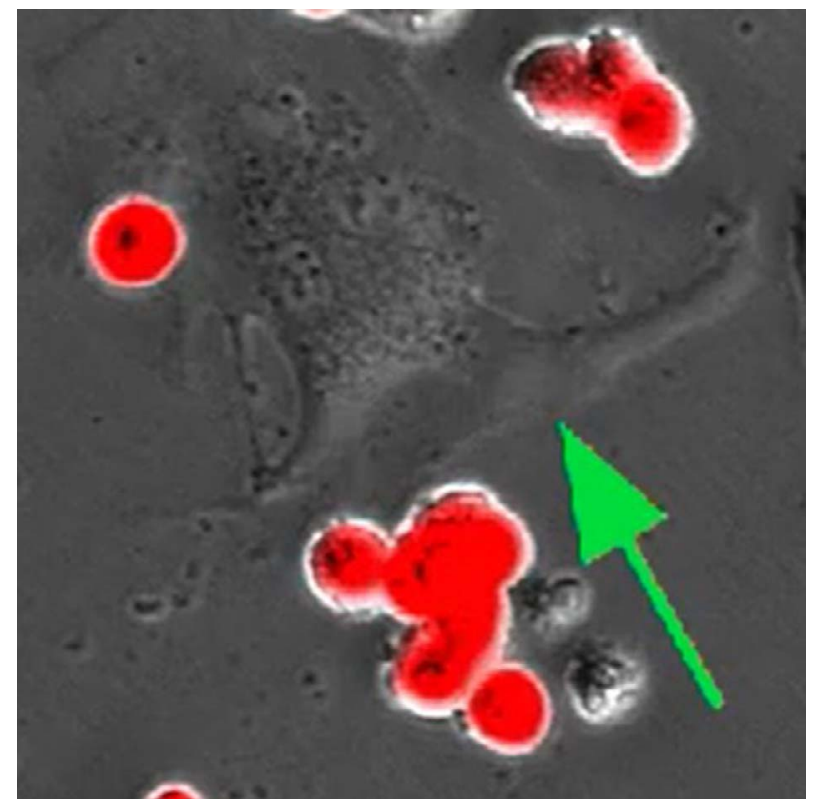

Video 2. The second video of co-culturing MGC803 cells with HMVEC cells. Another time-lapse video with the same conditions as Video 1. Intercellular ACE2-RFP transfer from cancer cells into endothelial cells (green arrows) was not observed. https://vcm. edpsciences.org/10.1051/vcm/20210003\#V2 


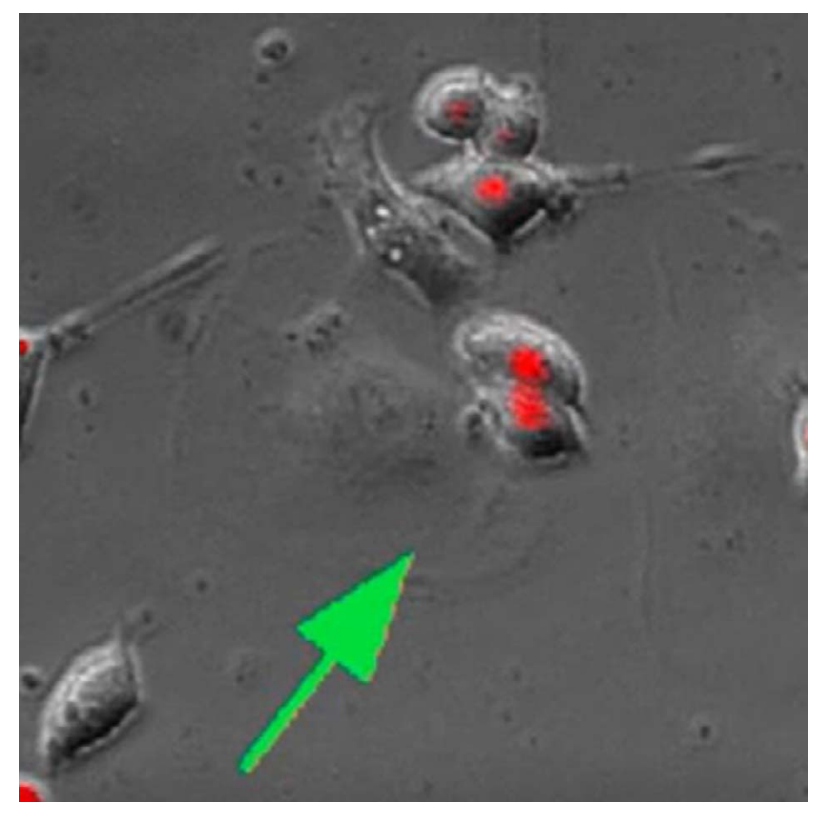

Video 3. Co-culturing Capan-2 pancreatic cancer cells with HMVEC cells. A time-lapse video demonstrating the movement of fusion protein ACE2-RFP (red) in cancer cells. There was no clear and convincing moment demonstrating ACE2-RFP transfer from cancer cells into endothelial cells (green arrows). https://vcm. edpsciences.org/10.1051/vcm/20210003\#V3

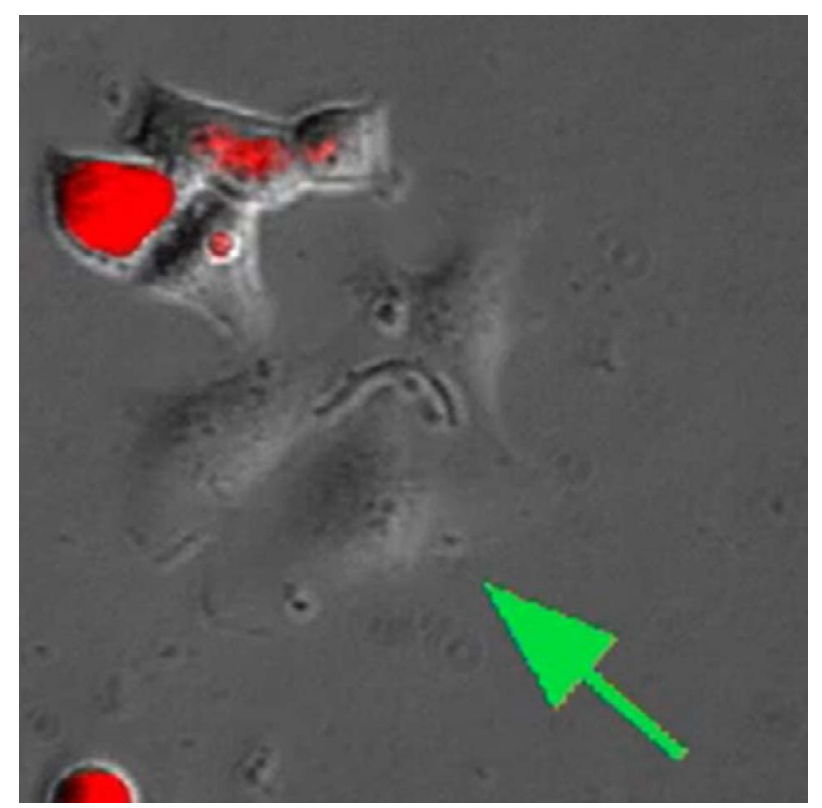

Video 4. Another video of co-culturing Capan-2 cells with HMVEC cells. A second video with the same conditions as Video 3. There was no clear and convincing moment demonstrating ACE2-RFP transfer from cancer cells into endothelial cells (green arrows). https://vcm.edpsciences.org/10.1051/vcm/20210003\#V4

COVID-19 patients without cancers [7, 19]. A recent study showed that 13 common cancers all had an increased risk of SARS-CoV-2 infection than patients without cancer, especially for those with blood cancer [20]. Moreover, newly diagnosed cancer patients are the most vulnerable among all cancer patients.

Consistent with the bioinformatics' analysis that ACE2 mRNA was aberrantly expressed in many types of tumors [3], the elevated expression of ACE2 in the majority of tumor tissues found in our study might provide a clue to explaining this clinical phenomenon. Tumor cells over-expressing ACE2 have been proposed to provide the entrance for SARS-CoV-2 infection, and this entrance has been suspected to be shared between cancer cells and endothelial cells. However, when co-culturing HMVEC with human cancer cells overexpressing the ACE2-RFP fusion protein, we did not observe ACE2 transferring from tumor cells into endothelial cells.

The prognostic role of ACE2 in cancer patients without COVID-19 has been preliminarily evaluated. Elevated ACE2 expression in tumor tissues correlates with better patient prognosis in cancers of the liver [21], kidney [21], uterine endometrium [4], and the breast [5].

As to the mechanism underlying ACE2 aberrant expression, bioinformatics found neither mutations nor DNA copy variation was correlated with the overexpression of ACE2 [3]. In contrast, hypo DNA methylation of ACE2 has been found to be inversely correlated with ACE2 overexpression in cancer cells [3]. A reduced DNA methylation level is a possible reason for ACE2 overexpression in colon adenocarcinoma, rectal adenocarcinoma, pancreatic adenocarcinoma, rectum adenocarcinoma, and lung adenocarcinoma. While an increased DNA methylation level might be the cause of ACE2 being downregulated in testicular germ cell tumors.

Deteriorating factors of cancer patients with COVID-19 include being male, elderly, having more comorbidities, a history of smoking, cardiovascular disease, type 2 diabetes, obesity, asthma, radiotherapy, chemotherapy, and immunotherapy $[19,22,23]$. It is therefore believed that the impaired immune system in cancer patients might be a common reason for the increased risk of COVID-19 infection.

However, in the present study, we only applied short-term in vitro co-culture of the cells. Long-term cell culture using more types of endothelial cells as well as in vivo experiments are warranted to exclude the possibility of ACE2 transferring from tumor cells to endothelial cells.

Taken together, we found that ACE2 expression was upregulated in colorectal adenocarcinoma, pancreatic adenocarcinoma, gastric adenocarcinoma, and papillary renal cell carcinoma. While it was downregulated in chromophobe renal cell carcinoma, testicular germ cell tumors, and papillary thyroid carcinoma. Only half of the tumor vasculature expressed ACE2. Intercellular exchange of ACE2 from cancer cells into endothelial cells was not observed in our short-term cell co-culture.

\section{Conflict of interest}

The authors declare that they do not have any conflict of interest.

Acknowledgements. This work was partly supported by grants from the National Natural Science Foundation of China (No. 82073220, 
No. 81872384, and No. 81672872 to C.Q., No. 81972785, No. 81773162 , and No. 81572901 to B.H.), and a research program from Sun Yat-sen University (No. 84000-18843409 to C.Q.).

\section{References}

1. Jia H, Pulmonary Angiotensin-Converting Enzyme 2 (ACE2) and inflammatory lung disease. Shock. 2016;46(3):239-248.

2. Chen YY, Zhang P, Zhou XM, et al. Relationship between genetic variants of ACE2 gene and circulating levels of ACE2 and its metabolites. J Clin Pharm Ther. 2018;43(2):189-195.

3. Chai $\mathrm{P}, \mathrm{Yu} \mathrm{J}, \mathrm{Ge} \mathrm{S}$, et al. Genetic alteration, RNA expression, and DNA methylation profiling of coronavirus disease 2019 (COVID-19) receptor ACE2 in malignancies: a pan-cancer analysis. J Hematol Oncol. 2020;13(1):43.

4. Yang J, Li H, Hu S, et al. ACE2 correlated with immune infiltration serves as a prognostic biomarker in endometrial carcinoma and renal papillary cell carcinoma: implication for COVID-19. Aging (Albany NY). 2020;12(8):6518-6535.

5. Zhang Q, Lu S, Li T, et al. ACE2 inhibits breast cancer angiogenesis via suppressing the VEGFa/VEGFR2/ERK pathway. J Exp Clin Cancer Res. 2019;38(1):173.

6. Addeo A, Friedlaender A. Cancer and COVID-19: Unmasking their ties. Cancer Treat Rev. 2020;88, 102041.

7. Liang W, Guan W, Chen R, et al. Cancer patients in SARSCoV-2 infection: a nationwide analysis in China. Lancet Oncol. 2020;21(3):335-337.

8. Pons S, Fodil S, Azoulay E, et al. The vascular endothelium: the cornerstone of organ dysfunction in severe SARS-CoV-2 infection. Crit Care. 2020;24(1):353.

9. Brown K, Fidanboylu M, Wong W. Intercellular exchange of surface molecules and its physiological relevance. Arch Immunol Ther Exp. 2010;58(4):263-272.

10. Sakurai T, Woolls MJ, Jin SW, et al. Inter-cellular exchange of cellular components via VE-cadherin-dependent transendocytosis. PLoS One. 2014;9(6):e90736.

11. Burtey A, Wagner M, Hodneland E, et al. Intercellular transfer of transferrin receptor by a contact-, Rab8-dependent mechanism involving tunneling nanotubes. FASEB J. 2015;29(11): 4695-4712.
12. Zhu W, Cai MY, Tong ZT, et al. Overexpression of EIF5A2 promotes colorectal carcinoma cell aggressiveness by upregulating MTA1 through C-myc to induce epithelial-mesenchymaltransition. Gut. 2012;61(4):562-575.

13. Li P, He HQ, Zhu CM, et al. The prognostic significance of lymphovascular invasion in patients with resectable gastric cancer: a large retrospective study from Southern China. BMC Cancer. 2015;15:370.

14. Li XJ, Ong CK, Cao Y, et al. Serglycin is a theranostic target in nasopharyngeal carcinoma that promotes metastasis. Cancer Res. 2011;71(8):3162-3172.

15. Li XJ, Peng LX, Shao JY, et al. As an independent unfavorable prognostic factor, IL-8 promotes metastasis of nasopharyngeal carcinoma through induction of epithelial-mesenchymal transition and activation of AKT signaling. Carcinogenesis. 2012;33(7): 1302-1309.

16. Xie P, Yang JP, Cao Y, et al. Promoting tumorigenesis in nasopharyngeal carcinoma, NEDD8 serves as a potential theranostic target. Cell Death Dis. 2017;8(6):e2834.

17. Lu R, Zhao X, Li J, et al. Genomic characterisation and epidemiology of 2019 novel coronavirus: implications for virus origins and receptor binding. Lancet. 2020;395(10224):565-574.

18. Wan Y, Shang J, Graham R, et al. Receptor recognition by the novel coronavirus from Wuhan: an analysis based on decade-long structural studies of SARS coronavirus. J Virol. 2020;94(7): e00127-20.

19. Yu J, Ouyang W, Chua MLK, et al. SARS-CoV-2 transmission in patients with cancer at a tertiary care hospital in Wuhan, China. JAMA Oncol. 2020;6(7):1108-1110.

20. Wang QQ, Berger NA, Xu R. Analyses of risk, racial disparity, and outcomes among US patients with cancer and COVID-19 infection. JAMA Oncol. 2021;7(2):220-227.

21. Fu J, Zhou B, Zhang L, et al. Expressions and significances of the angiotensin-converting enzyme 2 gene, the receptor of SARSCoV-2 for COVID-19. Mol Biol Rep. 2020;47(6):4383-4392.

22. Kuderer NM, Choueiri TK, Shah DP, et al. Clinical impact of COVID-19 on patients with cancer (CCC19): a cohort study. Lancet. 2020;395(10241):P1907-P1918.

23. Lee LYW, Cazier JB, Angelis V, et al. COVID-19 mortality in patients with cancer on chemotherapy or other anticancer treatments: a prospective cohort study. Lancet. 2020;395(10241): P1919-P1926.

Cite this article as: Liu Z-J, Mei Y, Lu J-L, Lu J-B, Cao Y, Cai M-Y, Zheng L-S, Wang M-D, Peng L-X, Li Y, Huang B-J, Yun J-P \& Qian C-N. ACE2 in tumor cells and tumor vasculature: Negligible intercellular transfer from cancer cells into endothelial cells. Visualized Cancer Medicine. 2021; $2,3$. 